\title{
Existence and approximations of fixed points for contractive mappings of integral type
}

Zeqing Liu' ${ }^{1}$ Xiaozhu Li and Shin Min Kang ${ }^{2 *}$

\section{${ }^{*}$ Correspondence:}

smkang@gnu.ac.kr

${ }^{2}$ Department of Mathematics and

RINS, Gyeongsang National

University, Jinju, 660-701, Korea

Full list of author information is

available at the end of the article

\section{Springer}

\begin{abstract}
The existence, uniqueness, and iterative approximations of fixed points for four classes of contractive mappings of integral type in complete metric spaces are established. The results presented in this paper generalize indeed several results of Branciari (J. Math. Math. Sci. 29(9):531-536, 2002), Rhoades (Int. J. Math. Math. Sci. 2003(63):4007-4013, 2003) and Liu et al. (Fixed Point Theory Appl. 2011:64, 2011). Four illustrative examples with uncountably many points are also included.
\end{abstract}

MSC: $54 \mathrm{H} 25$

Keywords: contractive mappings of integral type; fixed point; complete metric space

\section{Introduction}

Over the past decade the researchers [1-18] introduced a lot of contractive mappings of integral type and discussed the existence of fixed points and common fixed points for these mappings in metric spaces and modular spaces, respectively. Branciari [5] was the first to study the existence of fixed points for the contractive mapping of integral type and proved the following result, which extends the Banach fixed point theorem.

Theorem 1.1 ([5]) Let $f$ be a mapping from a complete metric space $(X, d)$ into itself satisfying

$$
\int_{0}^{d(f x, f y)} \varphi(t) d t \leq c \int_{0}^{d(x, y)} \varphi(t) d t, \quad \forall x, y \in X,
$$

where $c \in(0,1)$ is a constant and $\varphi \in \Phi=\left\{\varphi: \varphi: \mathbb{R}^{+} \rightarrow \mathbb{R}^{+}\right.$is Lebesgue integrable, summable on each compact subset of $\mathbb{R}^{+}$and $\int_{0}^{\varepsilon} \varphi(t) d t>0$ for each $\left.\varepsilon>0\right\}$.

Then $f$ has a unique fixed point $a \in X$ such that $\lim _{n \rightarrow \infty} f^{n} x=a$ for each $x \in X$.

Rhoades [16] and Liu et al. [10] extended the result of Branciari and proved the following fixed point theorems.

Theorem $1.2([16])$ Let $f$ be a mapping from a complete metric space $(X, d)$ into itself satisfying

$$
\int_{0}^{d\left(f x_{x} f y\right)} \varphi(t) d t \leq c \int_{0}^{\max \left\{d(x, y), d\left(x_{f} f x\right), d(y, f y), \frac{1}{2}\left[d\left(x_{x} f y\right)+d(y, f x)\right]\right\}} \varphi(t) d t, \quad \forall x, y \in X,
$$

(0) 2014 Liu et al.; licensee Springer. This is an Open Access article distributed under the terms of the Creative Commons Attribution License (http://creativecommons.org/licenses/by/2.0), which permits unrestricted use, distribution, and reproduction in any medium, provided the original work is properly cited. 
where $c \in(0,1)$ is a constant and $\varphi \in \Phi$. Then $f$ has a unique fixed point $a \in X$ such that $\lim _{n \rightarrow \infty} f^{n} x=$ a for each $x \in X$.

Theorem 1.3 ([16]) Let $f$ be a mapping from a complete metric space $(X, d)$ into itself satisfying

$$
\int_{0}^{d(f x, f y)} \varphi(t) d t \leq c \int_{0}^{\max \{d(x, y), d(x, f x), d(y, f y), d(x, f y), d(y, f x)\}} \varphi(t) d t, \quad \forall x, y \in X,
$$

where $c \in(0,1)$ is a constant and $\varphi \in \Phi$. Assume that $f$ has a bounded orbit at some point $x \in X$. Then $f$ has a unique fixed point $a \in X$ such that $\lim _{n \rightarrow \infty} f^{n} x=a$.

Theorem 1.4 ([10]) Let $f$ be a mapping from a complete metric space $(X, d)$ into itself satisfying

$$
\int_{0}^{d\left(f x_{2} f y\right)} \varphi(t) d t \leq \alpha(d(x, y)) \int_{0}^{d(x, y)} \varphi(t) d t, \quad \forall x, y \in X
$$

where $\varphi \in \Phi$ and $\alpha: \mathbb{R}^{+} \rightarrow[0,1)$ is a function with

$$
\limsup _{s \rightarrow t} \alpha(s)<1, \quad \forall t>0 .
$$

Then $f$ has a unique fixed point $a \in X$ such that $\lim _{n \rightarrow \infty} f^{n} x=a$ for each $x \in X$.

Theorem 1.5 ([10]) Let $f$ be a mapping from a complete metric space $(X, d)$ into itself satisfying

$$
\int_{0}^{d(f x, f y)} \varphi(t) d t \leq \alpha(d(x, y)) \int_{0}^{d(x, f x)} \varphi(t) d t+\beta(d(x, y)) \int_{0}^{d(y, f y)} \varphi(t) d t, \quad \forall x, y \in X,
$$

where $\varphi \in \Phi$ and $\alpha, \beta: \mathbb{R}^{+} \rightarrow[0,1)$ are two functions with

$$
\alpha(t)+\beta(t)<1, \quad \forall t \in \mathbb{R}^{+}, \quad \limsup _{s \rightarrow 0^{+}} \beta(s)<1, \quad \limsup _{s \rightarrow t^{+}} \frac{\alpha(s)}{1-\beta(s)}<1, \quad \forall t>0 .
$$

Then $f$ has a unique fixed point $a \in X$ such that $\lim _{n \rightarrow \infty} f^{n} x=a$ for each $x \in X$.

The purposes of this paper are both to study the existence, uniqueness, and iterative approximations of fixed points for four new classes of contractive mappings of integral type, which include the contractive mappings of integral type in $[5,10,16]$ as special cases, and to construct four examples with uncountably many points to illustrate that the results obtained properly generalize Theorems 1.1-1.5 or are different from these theorems.

\section{Preliminaries}

Throughout this paper, we assume that $\mathbb{R}=(-\infty,+\infty), \mathbb{R}^{+}=[0,+\infty), \mathbb{N}_{0}=\{0\} \cup \mathbb{N}$, where $\mathbb{N}$ denotes the set of all positive integers. Let $(X, d)$ be a metric space. For $f: X \rightarrow X, A \subset X$ and $(x, y, n) \in X^{2} \times \mathbb{N}_{0}$, put

$$
\begin{aligned}
& O_{f}(x, n)=\left\{f^{i} x: 0 \leq i \leq n\right\}, O_{f}(x)=\left\{f^{i} x: \forall i \in \mathbb{N}_{0}\right\}, \\
& d_{n}=d\left(f^{n} x, f^{n+1} x\right), \delta(A)=\sup \{d(u, v): \forall u, v \in A\},
\end{aligned}
$$




$$
\begin{aligned}
& m_{1}(x, y)=\max \left\{d(x, y), d(x, f x), d(y, f y), \frac{1}{2}[d(x, f y)+d(y, f x)]\right\}, \\
& m_{2}(x, y)=\max \{d(x, y), d(x, f x), d(y, f y), d(x, f y), d(y, f x)\} .
\end{aligned}
$$

The $O_{f}(x)$ and $O_{f}(x, n)$ are called the orbit and $n$th orbit of $f$ at $x$, respectively.

Let

$\Psi_{1}=\left\{\alpha: \alpha: \mathbb{R}^{+} \rightarrow[0,1)\right.$ is a function with $\left.\limsup _{s \rightarrow t} \alpha(s)<1, \forall t \in \mathbb{R}^{+}\right\}$,

$\Psi_{2}=\left\{\alpha: \alpha: \mathbb{R}^{+} \rightarrow[0,1)\right.$ is a function with $\left.\limsup _{s \rightarrow t} \alpha(s)<1, \forall t>0\right\}$,

$\Psi_{3}=\left\{\alpha: \alpha: \mathbb{R}^{+} \rightarrow[0,1)\right.$ is a function such that $\sup \{\alpha(s): \forall s \in B\}<1$ for each nonempty bounded subset $B$ in $\mathbb{R}^{+}$.

The following lemma plays an important role in this paper.

Lemma $2.1([10])$ Let $\varphi \in \Phi$ and $\left\{r_{n}\right\}_{n \in \mathbb{N}}$ be a nonnegative sequence with $\lim _{n \rightarrow \infty} r_{n}=a$.

Then

$$
\lim _{n \rightarrow \infty} \int_{0}^{r_{n}} \varphi(t) d t=\int_{0}^{a} \varphi(t) d t
$$

\section{Four fixed point theorems}

In this section we show the existence, uniqueness and iterative approximations of fixed points for four classes of contractive mappings of integral type.

Theorem 3.1 Let $f$ be a mapping from a complete metric space $(X, d)$ into itself satisfying

$$
\int_{0}^{d(f x, f y)} \varphi(t) d t \leq \alpha(d(x, y)) \int_{0}^{m_{1}(x, y)} \varphi(t) d t, \quad \forall x, y \in X,
$$

where $(\varphi, \alpha) \in \Phi \times \Psi_{1}$. Then $f$ has a unique fixed point $a \in X$ such that $\lim _{n \rightarrow \infty} f^{n} x=a$ for each $x \in X$.

Proof Let $x$ be an arbitrary point in $X$. Note that

$$
\begin{aligned}
m_{1}\left(f^{n-1} x, f^{n} x\right)= & \max \left\{d\left(f^{n-1} x, f^{n} x\right), d\left(f^{n-1} x, f^{n} x\right), d\left(f^{n} x, f^{n+1} x\right),\right. \\
& \left.\frac{1}{2}\left[d\left(f^{n-1} x, f^{n+1} x\right)+d\left(f^{n} x, f^{n} x\right)\right]\right\} \\
= & \max \left\{d\left(f^{n-1} x, f^{n} x\right), d\left(f^{n} x, f^{n+1} x\right)\right\} \\
= & \max \left\{d_{n-1}, d_{n}\right\}, \quad \forall n \in \mathbb{N} .
\end{aligned}
$$

It follows from (3.1) and (3.2) that

$$
\begin{aligned}
\int_{0}^{d_{n}} \varphi(t) d t & =\int_{0}^{d\left(f^{n} x f^{n+1} x\right)} \varphi(t) d t \\
& \leq \alpha\left(d\left(f^{n-1} x, f^{n} x\right)\right) \int_{0}^{\left.m_{1} f^{n-1} x f^{n} x\right)} \varphi(t) d t \\
& \leq \alpha\left(d_{n-1}\right) \int_{0}^{\max \left\{d_{n-1}, d_{n}\right\}} \varphi(t) d t, \quad \forall n \in \mathbb{N} .
\end{aligned}
$$

Now we prove that

$$
d_{n} \leq d_{n-1}, \quad \forall n \in \mathbb{N} .
$$


Suppose that (3.4) does not hold. That is, there exists some $n_{0} \in \mathbb{N}$ satisfying

$$
d_{n_{0}}>d_{n_{0}-1}
$$

Since $\varphi \in \Phi$ and $\alpha\left(\mathbb{R}^{+}\right) \subseteq[0,1)$, it follows from (3.3) and (3.5) that

$$
0<\int_{0}^{d_{n_{0}}} \varphi(t) d t \leq \alpha\left(d_{n_{0}-1}\right) \int_{0}^{\max \left\{d_{n_{0}-1}, d_{n_{0}}\right\}} \varphi(t) d t<\int_{0}^{d_{n_{0}}} \varphi(t) d t
$$

which is a contradiction and hence (3.4) holds. Clearly, (3.4) implies that there exists a constant $c$ with $\lim _{n \rightarrow \infty} d_{n}=c \geq 0$.

Next we prove that $c=0$. Otherwise $c>0$. Taking the upper limit in (3.3) and using Lemma 2.1 and $\varphi \in \Phi$, we conclude that

$$
\begin{aligned}
0 & <\int_{0}^{c} \varphi(t) d t=\limsup _{n \rightarrow \infty} \int_{0}^{d_{n}} \varphi(t) d t \\
& \leq \limsup _{n \rightarrow \infty}\left(\alpha\left(d_{n-1}\right) \int_{0}^{\max \left\{d_{n-1}, d_{n}\right\}} \varphi(t) d t\right) \\
& \leq \limsup _{n \rightarrow \infty} \alpha\left(d_{n-1}\right) \cdot \limsup _{n \rightarrow \infty} \int_{0}^{d_{n-1}} \varphi(t) d t \\
& \leq\left(\limsup _{s \rightarrow c} \alpha(s)\right) \int_{0}^{c} \varphi(t) d t<\int_{0}^{c} \varphi(t) d t,
\end{aligned}
$$

which is absurd. Therefore, $c=0$, that is,

$$
\lim _{n \rightarrow \infty} d_{n}=0 .
$$

Now we claim that $\left\{f^{n} x\right\}_{n \in \mathbb{N}}$ is a Cauchy sequence. Suppose that $\left\{f^{n} x\right\}_{n \in \mathbb{N}}$ is not a Cauchy sequence, which means that there is a constant $\varepsilon>0$ such that for each positive integer $k$, there are positive integers $m(k)$ and $n(k)$ with $m(k)>n(k)>k$ such that

$$
d\left(f^{m(k)} x, f^{n(k)} x\right)>\varepsilon
$$

For each positive integer $k$, let $m(k)$ denote the least integer exceeding $n(k)$ and satisfying the above inequality. It follows that

$$
d\left(f^{m(k)} x, f^{n(k)} x\right)>\varepsilon \quad \text { and } \quad d\left(f^{m(k)-1} x, f^{n(k)} x\right) \leq \varepsilon, \quad \forall k \in \mathbb{N} .
$$

Note that

$$
\begin{aligned}
& d\left(f^{m(k)} x, f^{n(k)} x\right) \leq d\left(f^{n(k)} x, f^{m(k)-1} x\right)+d_{m(k)-1}, \quad \forall k \in \mathbb{N} ; \\
& \left|d\left(f^{m(k)} x, f^{n(k)+1} x\right)-d\left(f^{m(k)} x, f^{n(k)} x\right)\right| \leq d_{n(k)}, \quad \forall k \in \mathbb{N} ; \\
& \left|d\left(f^{m(k)+1} x, f^{n(k)+1} x\right)-d\left(f^{m(k)} x, f^{n(k)+1} x\right)\right| \leq d_{m(k)}, \quad \forall k \in \mathbb{N} ; \\
& \left|d\left(f^{m(k)+1} x, f^{n(k)+1} x\right)-d\left(f^{m(k)+1} x, f^{n(k)+2} x\right)\right| \leq d_{n(k)+1}, \quad \forall k \in \mathbb{N} ; \\
& \left|d\left(f^{m(k)} x, f^{n(k)+2} x\right)-d\left(f^{m(k)} x, f^{n(k)+1} x\right)\right| \leq d_{n(k)+1}, \quad \forall k \in \mathbb{N} .
\end{aligned}
$$


Making use of (3.6)-(3.8), we obtain

$$
\begin{aligned}
\varepsilon & =\lim _{k \rightarrow \infty} d\left(f^{n(k)} x, f^{m(k)} x\right) \\
& =\lim _{k \rightarrow \infty} d\left(f^{m(k)} x, f^{n(k)+1} x\right) \\
& =\lim _{k \rightarrow \infty} d\left(f^{m(k)+1} x, f^{n(k)+1} x\right) \\
& =\lim _{k \rightarrow \infty} d\left(f^{m(k)+1} x, f^{n(k)+2} x\right) \\
& =\lim _{k \rightarrow \infty} d\left(f^{m(k)} x, f^{n(k)+2} x\right) .
\end{aligned}
$$

It follows from (3.6) and (3.9) that

$$
\begin{aligned}
m_{1} & \left(f^{m(k)} x, f^{n(k)+1} x\right) \\
= & \max \left\{d\left(f^{m(k)} x, f^{n(k)+1} x\right), d\left(f^{m(k)} x, f^{m(k)+1} x\right), d\left(f^{n(k)+1} x, f^{n(k)+2} x\right),\right. \\
& \left.\frac{1}{2}\left[d\left(f^{m(k)} x, f^{n(k)+2} x\right)+d\left(f^{n(k)+1} x, f^{m(k)+1} x\right)\right]\right\} \\
\rightarrow & \max \{\varepsilon, 0,0, \varepsilon\}=\varepsilon \quad \text { as } k \rightarrow \infty,
\end{aligned}
$$

which together with (3.1), Lemma 2.1, and $(\varphi, \alpha) \in \Phi \times \Psi_{1}$ gives

$$
\begin{aligned}
0 & <\int_{0}^{\varepsilon} \varphi(t) d t=\limsup _{k \rightarrow \infty} \int_{0}^{d\left(f^{m(k)+1} x, f^{n(k)+2} x\right)} \varphi(t) d t \\
& \leq \limsup _{k \rightarrow \infty}\left(\alpha\left(d\left(f^{m(k)} x, f^{n(k)+1} x\right)\right) \int_{0}^{m_{1}\left(f^{m(k)} x, f^{n(k)+1} x\right)} \varphi(t) d t\right) \\
& \leq \limsup _{k \rightarrow \infty} \alpha\left(d\left(f^{m(k)} x, f^{n(k)+1} x\right)\right) \cdot \limsup _{k \rightarrow \infty} \int_{0}^{m_{1}\left(f^{m(k)} x, f^{n(k)+1} x\right)} \varphi(t) d t \\
& \leq\left(\limsup _{s \rightarrow \varepsilon} \alpha(s)\right) \int_{0}^{\varepsilon} \varphi(t) d t<\int_{0}^{\varepsilon} \varphi(t) d t,
\end{aligned}
$$

which is a contradiction. Thus $\left\{f^{n} x\right\}_{n \in \mathbb{N}}$ is a Cauchy sequence. Since $(X, d)$ is a complete metric space, it follows that there exists a point $a \in X$ such that $\lim _{n \rightarrow \infty} f^{n} x=a$. Suppose that $f a \neq a$. It is clear that (3.6) implies that

$$
\begin{aligned}
m_{1}\left(f^{n} x, a\right) & =\max \left\{d\left(f^{n} x, a\right), d\left(f^{n} x, f^{n+1} x\right), d(a, f a), \frac{1}{2}\left[d\left(f^{n} x, f a\right)+d\left(a, f^{n+1} x\right)\right]\right\} \\
& \rightarrow d(a, f a) \quad \text { as } n \rightarrow \infty,
\end{aligned}
$$

which together with (3.1), Lemma 2.1, and $(\varphi, \alpha) \in \Phi \times \Psi_{1}$ yields

$$
\begin{aligned}
0 & <\int_{0}^{d(a, f a)} \varphi(t) d t=\limsup _{n \rightarrow \infty} \int_{0}^{d\left(f^{n+1} x, f a\right)} \varphi(t) d t \\
& \leq \limsup _{n \rightarrow \infty}\left(\alpha\left(d\left(f^{n} x, a\right)\right) \int_{0}^{m_{1}\left(f^{n} x, a\right)} \varphi(t) d t\right)
\end{aligned}
$$




$$
\begin{aligned}
& \leq \limsup _{n \rightarrow \infty} \alpha\left(d\left(f^{n} x, a\right)\right) \cdot \limsup _{n \rightarrow \infty} \int_{0}^{m_{1}\left(f^{n} x, a\right)} \varphi(t) d t \\
& \leq\left(\limsup _{s \rightarrow 0} \alpha(s)\right) \int_{0}^{d(a, f a)} \varphi(t) d t<\int_{0}^{d(a, f a)} \varphi(t) d t \quad \text { as } n \rightarrow \infty,
\end{aligned}
$$

which is a contradiction. That is, $a=f a$.

Finally, we prove that $a$ is a unique fixed point of $f$ in $X$. Suppose that $f$ has another fixed point $b \in X \backslash\{a\}$. Note that

$$
m_{1}(a, b)=\max \left\{d(a, b), d(a, f a), d(b, f b), \frac{1}{2}[d(a, f b)+d(b, f a)]\right\}=d(a, b) .
$$

It follows from (3.1), $\alpha\left(\mathbb{R}^{+}\right) \subseteq[0,1)$ and $\varphi \in \Phi$ that

$$
0<\int_{0}^{d(a, b)} \varphi(t) d t=\int_{0}^{d(f a, f b)} \varphi(t) d t \leq \alpha(d(a, b)) \int_{0}^{m_{1}(a, b)} \varphi(t) d t<\int_{0}^{d(a, b)} \varphi(t) d t,
$$

which is a contradiction. This completes the proof.

Theorem 3.2 Let $f$ be a mapping from a complete metric space $(X, d)$ into itself satisfying

$$
\int_{0}^{d\left(f x_{x} f y\right)} \varphi(t) d t \leq \alpha(d(x, y)) \int_{0}^{m_{2}(x, y)} \varphi(t) d t, \quad \forall x, y \in X
$$

where $(\varphi, \alpha) \in \Phi \times \Psi_{3}$. Assume that $f$ has a bounded orbit at some point $u \in X$. Then $f$ has a unique fixed point $a \in X$ such that $\lim _{n \rightarrow \infty} f^{n} u=a$.

Proof Without loss of generality we assume that $u \neq f u$. Now we prove that

$$
\text { for each } n \in \mathbb{N} \text { there exists } k \in \mathbb{N} \text { such that } k \leq n \text { and } \delta\left(O_{f}(u, n)\right)=d\left(u, f^{k} u\right) \text {. }
$$

Let $n \in \mathbb{N}$. It is clear that there exist $i, j \in \mathbb{N}_{0}$ such that $0 \leq i<j \leq n$ and $\delta\left(O_{f}(u, n)\right)=$ $d\left(f^{i} u, f^{j} u\right)$. Suppose that $\delta\left(O_{f}(u, n)\right)=d\left(f^{i} u, f^{j} u\right)$ for some $i, j \in \mathbb{N}$ with $0<i<j \leq n$. In light of (3.10) and $(\varphi, \alpha) \in \Phi \times \Psi_{3}$, we infer that

$$
\begin{aligned}
0 & <\int_{0}^{\delta\left(O_{f}(u, n)\right)} \varphi(t) d t=\int_{0}^{d\left(f^{i} u f f^{j} u\right)} \varphi(t) d t \\
& \leq \alpha\left(d\left(f^{i-1} u, f^{j-1} u\right)\right) \int_{0}^{m_{2}\left(f^{i-1} u f^{j-1} u\right)} \varphi(t) d t \\
& \leq \alpha\left(d\left(f^{i-1} u, f^{j-1} u\right)\right) \int_{0}^{\delta\left(O_{f}(u, n)\right)} \varphi(t) d t<\int_{0}^{\delta\left(O_{f}(u, n)\right)} \varphi(t) d t
\end{aligned}
$$

which is a contradiction. Thus (3.11) holds.

Next we prove that $O_{f}(u)$ is a Cauchy sequence. Suppose that $O_{f}(u)$ is not a Cauchy sequence. It follows that there exist an $\varepsilon>0$ and two strictly increasing sequences $\{m(p)\}_{p \in \mathbb{N}}$ and $\{n(p)\}_{p \in \mathbb{N}}$ with $m(p)>n(p)>p$ for each $p \in \mathbb{N}$ satisfying

$$
d\left(f^{m(p)} u, f^{n(p)} u\right)>\varepsilon, \quad \forall p \in \mathbb{N} .
$$


Put $r=\delta\left(O_{f}(u)\right)$ and $B=[0, r]$. Clearly $0<r<+\infty$. Observe that $(\varphi, \alpha) \in \Phi \times \Psi_{3}$ ensures that

$$
\lim _{p \rightarrow \infty}(\sup \{\alpha(s): s \in B\})^{n(p)} \int_{0}^{r} \varphi(t) d t=0,
$$

which implies that there exists some $p \in \mathbb{N}$ with

$$
(\sup \{\alpha(s): s \in B\})^{n(p)} \int_{0}^{r} \varphi(t) d t<\int_{0}^{\varepsilon} \varphi(t) d t .
$$

Using (3.10)-(3.13) and $(\varphi, \alpha) \in \Phi \times \Psi_{3}$, we know that there exist $0<k_{1} \leq m(p)-n(p)+1$, $0<k_{2} \leq m(p)-n(p)+2, \ldots$, and $0<k_{n(p)-1} \leq m(p)-1$ satisfying

$$
\begin{aligned}
\int_{0}^{\varepsilon} \varphi(t) d t \leq & \int_{0}^{d\left(f^{n(p)} u f^{m(p)} u\right)} \varphi(t) d t \\
\leq & \alpha\left(d\left(f^{n(p)-1} u, f^{m(p)-1} u\right)\right) \int_{0}^{m_{2}\left(f^{n(p)-1} u f^{m(p)-1} u\right)} \varphi(t) d t \\
\leq & \alpha\left(d\left(f^{n(p)-1} u, f^{m(p)-1} u\right)\right) \int_{0}^{\delta\left(O_{f}\left(f^{n(p)-1} u, m(p)-n(p)+1\right)\right)} \varphi(t) d t \\
= & \alpha\left(d\left(f^{n(p)-1} u, f^{m(p)-1} u\right)\right) \int_{0}^{d\left(f^{n(p)-1} u f^{k_{1}+n(p)-1} u\right)} \varphi(t) d t \\
\leq & \alpha\left(d\left(f^{n(p)-1} u, f^{m(p)-1} u\right)\right) \alpha\left(d\left(f^{n(p)-2} u, f^{k_{1}+n(p)-2} u\right)\right) \\
& \times \int_{0}^{\delta\left(O_{f}\left(f^{n(p)-2} u, k_{1}+1\right)\right)} \varphi(t) d t \\
= & \alpha\left(d\left(f^{n(p)-1} u, f^{m(p)-1} u\right)\right) \alpha\left(d\left(f^{n(p)-2} u, f^{k_{1}+n(p)-2} u\right)\right) \\
& \times \int_{0}^{d\left(f^{n(p)-2} u f^{k_{2}+n(p)-2} u\right)} \varphi(t) d t \\
\leq & \ldots \\
\leq & \alpha\left(d\left(f^{n(p)-1} u, f^{m(p)-1} u\right)\right) \alpha\left(d\left(f^{n(p)-2} u, f^{k_{1}+n(p)-2} u\right)\right) \cdots \alpha\left(d\left(u, f^{k_{n}(p)-1} u\right)\right) \\
& \times \int_{0}^{\delta\left(O_{f}(u, m(p))\right)} \varphi(t) d t \\
\leq & (\sup \{\alpha(s): s \in B\})^{n(p)} \int_{0}^{r} \varphi(t) d t \\
< & \int_{0}^{\varepsilon(t) d t,}
\end{aligned}
$$

which is impossible. Thus $\left\{f^{n} u\right\}_{n \in \mathbb{N}}$ is a Cauchy sequence. Since $(X, d)$ is complete, it follows that there exists $a \in X$ satisfying $\lim _{n \rightarrow \infty} f^{n} u=a$. Suppose that $d(a, f a)>0$. Note that

$$
\begin{aligned}
& \lim _{n \rightarrow \infty} m_{2}\left(f^{n} u, a\right) \\
& \quad=\lim _{n \rightarrow \infty} \max \left\{d\left(f^{n} u, a\right), d\left(f^{n} u, f^{n+1} u\right), d(a, f a), d\left(f^{n} u, f a\right), d\left(a, f^{n+1} u\right)\right\} \\
& =d(a, f a) .
\end{aligned}
$$


Taking the upper limit in (3.10) and using (3.14), Lemma 2.1, and $(\varphi, \alpha) \in \Phi \times \Psi_{3}$, we conclude that

$$
\begin{aligned}
0 & <\int_{0}^{d(a, f a)} \varphi(t) d t=\limsup _{n \rightarrow \infty} \int_{0}^{d\left(f^{n+1} u f f a\right)} \varphi(t) d t \\
& \leq \limsup _{n \rightarrow \infty}\left(\alpha\left(d\left(f^{n} u, a\right)\right) \int_{0}^{m_{2}\left(f^{n} u, a\right)} \varphi(t) d t\right) \\
& \leq \limsup _{n \rightarrow \infty} \alpha\left(d\left(f^{n} u, a\right)\right) \cdot \limsup _{n \rightarrow \infty} \int_{0}^{m_{2}\left(f^{n} u, a\right)} \varphi(t) d t \\
& \leq \sup \{\alpha(s): s \in[0,1]\} \int_{0}^{d\left(a_{f} f a\right)} \varphi(t) d t<\int_{0}^{d(a, f a)} \varphi(t) d t,
\end{aligned}
$$

which is absurd. Therefore, $d(a, f a)=0$, that is, $a=f a$.

Suppose that $f$ has another fixed point $w \in X \backslash\{a\}$. Since

$$
m_{2}(a, w)=\max \{d(a, w), d(a, f a), d(w, f w), d(a, f w), d(w, f a)\}=d(a, w),
$$

it follows from (3.10) that

$$
\begin{aligned}
0 & <\int_{0}^{d(a, w)} \varphi(t) d t \leq \alpha(d(a, w)) \int_{0}^{m_{2}(a, w)} \varphi(t) d t \\
& =\alpha(d(a, w)) \int_{0}^{d(a, w)} \varphi(t) d t<\int_{0}^{d(a, w)} \varphi(t) d t,
\end{aligned}
$$

which is a contradiction. That is, $f$ has a unique fixed point in $X$. This completes the proof.

As in the arguments of Theorems 3.1 and 3.2, we conclude similarly the following results and omit their proofs.

Theorem 3.3 Let $f$ be a mapping from a complete metric space $(X, d)$ into itself satisfying

$$
\int_{0}^{d\left(f x_{x} f y\right)} \varphi(t) d t \leq \alpha\left(m_{1}(x, y)\right) \int_{0}^{m_{1}(x, y)} \varphi(t) d t, \quad \forall x, y \in X
$$

where $(\varphi, \alpha) \in \Phi \times \Psi_{2}$. Then $f$ has a unique fixed point $a \in X$ such that $\lim _{n \rightarrow \infty} f^{n} x=$ a for each $x \in X$.

Theorem 3.4 Let $f$ be a mapping from a complete metric space $(X, d)$ into itself satisfying

$$
\int_{0}^{d\left(f x_{x} f y\right)} \varphi(t) d t \leq \alpha\left(m_{2}(x, y)\right) \int_{0}^{m_{2}(x, y)} \varphi(t) d t, \quad \forall x, y \in X
$$

where $(\varphi, \alpha) \in \Phi \times \Psi_{3}$. Assume that $f$ has a bounded orbit at some point $u \in X$. Then $f$ has a unique fixed point $a \in X$ such that $\lim _{n \rightarrow \infty} f^{n} u=a$.

\section{Remarks and illustrative examples}

Now we construct four examples with uncountably many points to show the fixed point theorems obtained in Section 3 generalize properly or are different from the known results in Section 1. 
Remark 4.1 Theorem 3.1 generalizes Theorem 1.2, which, in turns, extends Theorem 1.1. The following example proves that Theorem 3.1 both extends substantially Theorem 1.1 and is different from Theorem 1.4.

Example 4.2 Let $X=\left[0, \frac{3}{2}\right] \subset \mathbb{R}$ be endowed with the Euclidean metric $d=|\cdot|, f: X \rightarrow X$, $\varphi: \mathbb{R}^{+} \rightarrow \mathbb{R}^{+}$and $\alpha: \mathbb{R}^{+} \rightarrow[0,1)$ be defined by

$$
\begin{aligned}
& f(x)= \begin{cases}\frac{x}{3}, & \forall x \in[0,1], \\
x-1, & \forall x \in\left(1, \frac{3}{2}\right],\end{cases} \\
& \varphi(t)=2 t, \quad \forall t \in \mathbb{R}^{+} \text {and } \alpha(t)= \begin{cases}\frac{1}{3}, & t=0, \\
\frac{1}{2+t}, & \forall t \in(0,+\infty) .\end{cases}
\end{aligned}
$$

Obviously, $(\varphi, \alpha) \in \Phi \times \Psi_{1}$. Let $x, y \in X$ with $y<x$. In order to verify (3.1), we have to consider six possible cases as follows:

Case $1.1<y<x \leq \frac{3}{2}$. It is clear that

$$
\begin{aligned}
m_{1}(x, y) & =\max \left\{d(x, y), d(x, f x), d(y, f y), \frac{1}{2}[d(x, f y)+d(y, f x)]\right\} \\
& =\max \{x-y, 1,1,1\} \\
& =1=d(x, f x)
\end{aligned}
$$

and

$$
\begin{aligned}
\int_{0}^{d(f x, f y)} \varphi(t) d t & =(x-y)^{2} \leq \frac{1}{4}<\frac{2}{5} \leq \frac{1}{2+x-y}=\alpha(d(x, y)) \int_{0}^{1} \varphi(t) d t \\
& =\alpha(d(x, y)) \int_{0}^{m_{1}(x, y)} \varphi(t) d t .
\end{aligned}
$$

Case 2. $0 \leq y<\frac{x}{3}$ and $x \leq 1$. Note that

$$
\begin{aligned}
m_{1}(x, y) & =\max \left\{d(x, y), d(x, f x), d(y, f y), \frac{1}{2}[d(x, f y)+d(y, f x)]\right\} \\
& =\max \left\{x-y, \frac{2}{3} x, \frac{2}{3} y, \frac{2}{3}(x-y)\right\} \\
& =x-y=d(x, y)
\end{aligned}
$$

and

$$
\begin{aligned}
\int_{0}^{d(f x, f y)} \varphi(t) d t & =\left(\frac{x}{3}-\frac{y}{3}\right)^{2}=\frac{(x-y)^{2}}{9} \leq \frac{(x-y)^{2}}{2+x-y} \\
& =\alpha(d(x, y)) \int_{0}^{d(x, y)} \varphi(t) d t \\
& =\alpha(d(x, y)) \int_{0}^{m_{1}(x, y)} \varphi(t) d t
\end{aligned}
$$


Lu et al. Fixed Point Theory and Applications 2014, 2014:138

Page 10 of 20

http://www.fixedpointtheoryandapplications.com/content/2014/1/138

Case 3. $\frac{x}{3} \leq y<x \leq 1$. It follows that

$$
\begin{aligned}
m_{1}(x, y) & =\max \left\{d(x, y), d(x, f x), d(y, f y), \frac{1}{2}[d(x, f y)+d(y, f x)]\right\} \\
& =\max \left\{x-y, \frac{2}{3} x, \frac{2}{3} y, \frac{1}{2}\left(x-\frac{y}{3}+y-\frac{x}{3}\right)\right\} \\
& =\frac{2}{3} x=d(x, f x)
\end{aligned}
$$

and

$$
\begin{aligned}
\int_{0}^{d(f x, f y)} \varphi(t) d t & =\left(\frac{x}{3}-\frac{y}{3}\right)^{2}=\frac{(x-y)^{2}}{9} \\
& \leq \frac{1}{2+x-y} \cdot \frac{4}{9} x^{2} \\
& =\alpha(d(x, y)) \int_{0}^{d(x, f x)} \varphi(t) d t \\
& =\alpha(d(x, y)) \int_{0}^{m_{1}(x, y)} \varphi(t) d t
\end{aligned}
$$

Case 4. $\frac{1}{2}<y \leq 1<x \leq \frac{3}{2}$. Notice that

$$
\begin{aligned}
m_{1}(x, y) & =\max \left\{d(x, y), d(x, f x), d(y, f y), \frac{1}{2}[d(x, f y)+d(y, f x)]\right\} \\
& =\max \left\{x-y, 1, \frac{2}{3} y, \frac{1}{2}\left(1+\frac{2}{3} y\right)\right\} \\
& =1=d(x, f x)
\end{aligned}
$$

and

$$
\begin{aligned}
\int_{0}^{d(f x, f y)} \varphi(t) d t & =\left|x-1-\frac{y}{3}\right|^{2} \\
& \leq \frac{1}{9}<\frac{1}{3} \leq \frac{1}{2+x-y} \\
& =\alpha(d(x, y)) \int_{0}^{d(x, f x)} \varphi(t) d t \\
& =\alpha(d(x, y)) \int_{0}^{m_{1}(x, y)} \varphi(t) d t
\end{aligned}
$$

Case $5 . x-1 \leq y \leq \frac{1}{2}$ and $1<x \leq \frac{3}{2}$. It is easy to see that

$$
\begin{aligned}
m_{1}(x, y) & =\max \left\{d(x, y), d(x, f x), d(y, f y), \frac{1}{2}[d(x, f y)+d(y, f x)]\right\} \\
& =\max \left\{x-y, 1, \frac{2}{3} y, \frac{1}{2}\left(1+\frac{2}{3} y\right)\right\} \\
& =1=d(x, f x)
\end{aligned}
$$


and

$$
\begin{aligned}
\int_{0}^{d(f x, f y)} \varphi(t) d t & =\left|x-1-\frac{y}{3}\right|^{2} \\
& \leq \frac{1}{4}<\frac{2}{7} \leq \frac{1}{2+x-y} \\
& =\alpha(d(x, y)) \int_{0}^{d(x, f x)} \varphi(t) d t \\
& =\alpha(d(x, y)) \int_{0}^{m_{1}(x, y)} \varphi(t) d t .
\end{aligned}
$$

Case 6. $0 \leq y<x-1$ and $1<x \leq \frac{3}{2}$. It is easy to verify that

$$
\begin{aligned}
m_{1}(x, y) & =\max \left\{d(x, y), d(x, f x), d(y, f y), \frac{1}{2}[d(x, f y)+d(y, f x)]\right\} \\
& =\max \left\{x-y, 1, \frac{2}{3} y, \frac{1}{2}\left(2 x-\frac{4}{3} y-1\right)\right\} \\
& =x-y=d(x, y)
\end{aligned}
$$

and

$$
\begin{aligned}
\int_{0}^{d(f x, f y)} \varphi(t) d t & =\left|x-1-\frac{y}{3}\right|^{2} \\
& \leq \frac{1}{4}<\frac{2}{7} \leq \frac{1}{2+x-y} \leq \frac{(x-y)^{2}}{2+x-y} \\
& =\alpha(d(x, y)) \int_{0}^{d(x, y)} \varphi(t) d t \\
& =\alpha(d(x, y)) \int_{0}^{m_{1}(x, y)} \varphi(t) d t .
\end{aligned}
$$

That is, (3.1) holds. It follows from Theorem 3.1 that $f$ has a unique fixed point $0 \in X$ and $\lim _{n \rightarrow \infty} f^{n} x=0$ for each $x \in X$. But we invoke neither Theorem 1.1 nor Theorem 1.4 to show that $f$ possesses a fixed point in $X$.

Suppose that $f$ satisfies the conditions of Theorem 1.1, that is, there exists $c \in(0,1)$ satisfying

$$
\begin{aligned}
\frac{49}{900} & =\left|\frac{11}{10}-1-\frac{1}{3}\right|^{2}=\int_{0}^{d\left(f \frac{11}{10}, f 1\right)} \varphi(t) d t \leq c \int_{0}^{d\left(\frac{11}{10}, 1\right)} \varphi(t) d t \\
& =c\left|\frac{11}{10}-1\right|^{2}=\frac{c}{100},
\end{aligned}
$$

which means that

$$
1<\frac{49}{9} \leq c<1
$$

which is a contradiction. 
Suppose that $f$ satisfies the conditions of Theorem 1.4, that is, there exists $\alpha \in \Psi_{2}$ satisfying

$$
\begin{aligned}
\frac{1}{100} & =\left|\frac{13}{10}-\frac{12}{10}\right|^{2}=\int_{0}^{d\left(f\left(\frac{13}{10}, \frac{12}{10}\right)\right.} \varphi(t) d t \leq \alpha\left(d\left(\frac{13}{10}, \frac{12}{10}\right)\right) \int_{0}^{d\left(\frac{13}{10}, \frac{12}{10}\right)} \varphi(t) d t \\
& =\alpha\left(\frac{1}{10}\right)\left|\frac{13}{10}-\frac{12}{10}\right|^{2}=\frac{1}{100} \alpha\left(\frac{1}{10}\right),
\end{aligned}
$$

which implies that

$$
1 \leq \alpha\left(\frac{1}{10}\right)<1
$$

which is a contradiction.

Remark 4.3 Theorem 3.2 is a generalization of Theorem 1.3. The below example demonstrates that Theorem 3.2 is different from Theorem 1.4.

Example 4.4 Let $X=\left[1, \frac{3}{2}\right] \cup\left[2, \frac{5}{2}\right] \cup[3,+\infty) \subset \mathbb{R}$ be endowed with the Euclidean metric $d=|\cdot|, f: X \rightarrow X, \varphi: \mathbb{R}^{+} \rightarrow \mathbb{R}^{+}$and $\alpha: \mathbb{R}^{+} \rightarrow[0,1)$ be defined by

$$
\begin{aligned}
& f(x)= \begin{cases}1, & \forall x \in\left[1, \frac{3}{2}\right], \\
x-1, & \forall x \in\left[2, \frac{5}{2}\right], \\
\frac{3}{2}, & \forall x \in[3,+\infty),\end{cases} \\
& \varphi(t)=2 t, \quad \forall t \in \mathbb{R}^{+} \text {and } \alpha(t)= \begin{cases}\frac{1}{2}, & t=0, \\
\frac{t^{2}}{(1+t)^{2}}, & \forall t \in(0,+\infty) .\end{cases}
\end{aligned}
$$

It is easy to see that $(\varphi, \alpha) \in \Phi \times \Phi_{3}$ and $O_{f}(u)$ is bounded for each $u \in X$. Let $x, y \in X$ with $y<x$. In order to verify (3.10), we have to consider six possible cases as follows:

Case $1.1 \leq y<x \leq \frac{3}{2}$. It is clear that

$$
\int_{0}^{d(f x, f y)} \varphi(t) d t=0 \leq \alpha(d(x, y)) \int_{0}^{m_{2}(x, y)} \varphi(t) d t
$$

Case 2. $2 \leq y<x \leq \frac{5}{2}$. Note that

$$
\begin{aligned}
m_{2}(x, y) & =\max \{d(x, y), d(x, f x), d(y, f y), d(x, f y), d(y, f x)\} \\
& =\max \{x-y, 1,1, x-y+1, y-x+1\} \\
& =x-y+1=d(x, f y)
\end{aligned}
$$

and

$$
\begin{aligned}
\int_{0}^{d(f x, f y)} \varphi(t) d t & =|x-1-y+1|^{2} \\
& =(x-y)^{2} \leq \frac{(x-y)^{2}(x-y+1)^{2}}{(x-y+1)^{2}}
\end{aligned}
$$




$$
\begin{aligned}
& =\alpha(d(x, y)) \int_{0}^{d(x, f y)} \varphi(t) d t \\
& =\alpha(d(x, y)) \int_{0}^{m_{2}(x, y)} \varphi(t) d t
\end{aligned}
$$

Case $3.1 \leq y \leq \frac{3}{2}$ and $2 \leq x \leq \frac{5}{2}$. It follows that

$$
\begin{aligned}
m_{2}(x, y) & =\max \{d(x, y), d(x, f x), d(y, f y), d(x, f y), d(y, f x)\} \\
& =\max \{x-y, 1, y-1, x-1,|y-(x-1)|\} \\
& =x-1=d(x, f y)
\end{aligned}
$$

and

$$
\begin{aligned}
\int_{0}^{d(f x, f y)} \varphi(t) d t & =|x-1-1|^{2} \\
& =(x-2)^{2} \leq \frac{(x-y)^{2}(x-1)^{2}}{(x-y+1)^{2}} \\
& =\alpha(d(x, y)) \int_{0}^{d(x, f y)} \varphi(t) d t \\
& =\alpha(d(x, y)) \int_{0}^{m_{2}(x, y)} \varphi(t) d t
\end{aligned}
$$

Case $4.3 \leq y<x<+\infty$. It is easy to see that

$$
\int_{0}^{d(f x, f y)} \varphi(t) d t=0 \leq \alpha(d(x, y)) \int_{0}^{m_{2}(x, y)} \varphi(t) d t
$$

Case 5. $2 \leq y \leq \frac{5}{2}$ and $x \geq 3$. It follows that

$$
\begin{aligned}
m_{2}(x, y) & =\max \{d(x, y), d(x, f x), d(y, f y), d(x, f y), d(y, f x)\} \\
& =\max \left\{x-y, x-\frac{3}{2}, 1, x-y+1, y-\frac{3}{2}\right\} \\
& =x-y+1=d(x, f y)
\end{aligned}
$$

and

$$
\begin{aligned}
\int_{0}^{d(f x, f y)} \varphi(t) d t & =\left|\frac{3}{2}-(y-1)\right|^{2} \\
& =\left(\frac{5}{2}-y\right)^{2} \leq \frac{(x-y)^{2}(x-y+1)^{2}}{(x-y+1)^{2}} \\
& =\alpha(d(x, y)) \int_{0}^{d(x, f y)} \varphi(t) d t \\
& =\alpha(d(x, y)) \int_{0}^{m_{2}(x, y)} \varphi(t) d t
\end{aligned}
$$


Case $6.1 \leq y \leq \frac{3}{2}$ and $x \geq 3$. It is clear that

$$
\begin{aligned}
m_{2}(x, y) & =\max \{d(x, y), d(x, f x), d(y, f y), d(x, f y), d(y, f x)\} \\
& =\max \left\{x-y, x-\frac{3}{2}, y-1, x-1, \frac{3}{2}-y\right\} \\
& =x-1=d(x, f y)
\end{aligned}
$$

and

$$
\begin{aligned}
\int_{0}^{d(f x, f y)} \varphi(t) d t & =\left|\frac{3}{2}-1\right|^{2}=\frac{1}{4} \leq \frac{(x-1)^{2}(x-y)^{2}}{(1+x-y)^{2}} \\
& =\alpha(d(x, y)) \int_{0}^{d(x, f y)} \varphi(t) d t \\
& =\alpha(d(x, y)) \int_{0}^{m_{2}(x, y)} \varphi(t) d t .
\end{aligned}
$$

That is, the conditions of Theorem 3.2 are fulfilled. It follows from Theorem 3.2 that $f$ has a unique fixed point $1 \in X$ and $\lim _{n \rightarrow \infty} f^{n} u=1$ for each $u \in X$. However, Theorem 1.4 is useless in guaranteeing the existence of a fixed point of $f$ in $X$. Suppose that $f$ satisfies the conditions of Theorem 1.4, that is, there exists $\alpha \in \Psi_{2}$ satisfying

$$
\begin{aligned}
\frac{1}{100} & =\left|\frac{21}{10}-2\right|^{2}=\int_{0}^{d\left(f^{\frac{21}{10}}, f 2\right)} \varphi(t) d t \leq \alpha\left(d\left(\frac{21}{10}, 2\right)\right) \int_{0}^{d\left(\frac{21}{10}, 2\right)} \varphi(t) d t \\
& =\alpha\left(\frac{1}{10}\right)\left|\frac{21}{10}-2\right|^{2}=\frac{1}{100} \alpha\left(\frac{1}{10}\right),
\end{aligned}
$$

which yields

$$
1 \leq \alpha\left(\frac{1}{10}\right)<1,
$$

which is impossible.

Remark 4.5 Theorem 3.3 extends Theorems 1.1 and 1.2. The example below is an application of Theorem 3.3 .

Example 4.6 Let $X=\mathbb{R}^{+}$be endowed with the Euclidean metric $d=|\cdot|, f: X \rightarrow X, \varphi$ : $\mathbb{R}^{+} \rightarrow \mathbb{R}^{+}$and $\alpha: \mathbb{R}^{+} \rightarrow[0,1)$ be defined by

$$
\begin{aligned}
& f(x)= \begin{cases}\frac{x}{2}, & \forall x \in[0,1), \\
\frac{x}{1+x}, & \forall x \in[1,+\infty),\end{cases} \\
& \varphi(t)=2 t, \quad \forall t \in \mathbb{R}^{+} \text {and } \alpha(t)= \begin{cases}\frac{1}{4}, & \forall t \in\left[0, \frac{1}{2}\right), \\
\frac{t}{1+t}, & \forall t \in\left[\frac{1}{2},+\infty\right) .\end{cases}
\end{aligned}
$$

Obviously, $(\varphi, \alpha) \in \Phi \times \Psi_{2}$. Let $x, y \in X$ with $y<x$. In order to verify (3.15), we have to consider five possible cases as follows: 
Case $1.1 \leq y<x<+\infty$. It follows that

$$
\begin{aligned}
m_{1}(x, y) & =\max \left\{d(x, y), d(x, f x), d(y, f y), \frac{1}{2}[d(x, f y)+d(y, f x)]\right\} \\
& =\max \left\{x-y, \frac{x^{2}}{1+x}, \frac{y^{2}}{1+y}, \frac{1}{2}\left(x-\frac{y}{1+y}+y-\frac{x}{1+x}\right)\right\} \\
& =\frac{x^{2}}{1+x}=d(x, f x)
\end{aligned}
$$

and

$$
\begin{aligned}
\int_{0}^{d(f x, f y)} \varphi(t) d t & =\left(\frac{x}{1+x}-\frac{y}{1+y}\right)^{2} \\
& =\frac{(x-y)^{2}}{(1+x)^{2}(1+y)^{2}} \leq \frac{1}{16}(x-y)^{2} \\
& \leq \frac{1}{16}\left(\frac{x^{2}}{1+x}\right)^{2} \leq \alpha\left(\frac{x^{2}}{1+x}\right)\left(\frac{x^{2}}{1+x}\right)^{2} \\
& =\alpha\left(m_{1}(x, y)\right) \int_{0}^{m_{1}(x, y)} \varphi(t) d t
\end{aligned}
$$

Case 2. $\frac{x}{2} \leq y<x<1$. It follows that

$$
\begin{aligned}
m_{1}(x, y) & =\max \left\{d(x, y), d(x, f x), d(y, f y), \frac{1}{2}[d(x, f y)+d(y, f x)]\right\} \\
& =\max \left\{x-y, \frac{x}{2}, \frac{y}{2}, \frac{1}{2}\left(x-\frac{y}{2}+y-\frac{x}{2}\right)\right\} \\
& =\frac{x}{2}=d(x, f x)
\end{aligned}
$$

and

$$
\begin{aligned}
\int_{0}^{d(f x, f y)} \varphi(t) d t & =\left(\frac{x}{2}-\frac{y}{2}\right)^{2}=\frac{1}{4}(x-y)^{2} \\
& \leq \frac{1}{4}\left(\frac{x}{2}\right)^{2}=\alpha\left(\frac{x}{2}\right)\left(\frac{x}{2}\right)^{2} \\
& =\alpha\left(m_{1}(x, y)\right) \int_{0}^{m_{1}(x, y)} \varphi(t) d t
\end{aligned}
$$

Case 3. $0 \leq y<\frac{x}{2}$ and $x<1$. It is clear that

$$
\begin{aligned}
m_{1}(x, y) & =\max \left\{d(x, y), d(x, f x), d(y, f y), \frac{1}{2}[d(x, f y)+d(y, f x)]\right\} \\
& =\max \left\{x-y, \frac{x}{2}, \frac{y}{2}, \frac{1}{2}\left(x-\frac{y}{2}+\frac{x}{2}-y\right)\right\} \\
& =x-y=d(x, y)
\end{aligned}
$$


and

$$
\begin{aligned}
\int_{0}^{d(f x, f y)} \varphi(t) d t & =\left(\frac{x}{2}-\frac{y}{2}\right)^{2}=\frac{1}{4}(x-y)^{2} \\
& \leq \alpha(x-y)(x-y)^{2} \\
& =\alpha\left(m_{1}(x, y)\right) \int_{0}^{m_{1}(x, y)} \varphi(t) d t .
\end{aligned}
$$

Case $4 . \frac{x}{1+x} \leq y<1$ and $x \geq 1$. Notice that

$$
\begin{aligned}
m_{1}(x, y) & =\max \left\{d(x, y), d(x, f x), d(y, f y), \frac{1}{2}[d(x, f y)+d(y, f x)]\right\} \\
& =\max \left\{x-y, \frac{x^{2}}{1+x}, \frac{y}{2}, \frac{1}{2}\left(x-\frac{y}{2}+y-\frac{x}{1+x}\right)\right\} \\
& =\frac{x^{2}}{1+x}=d(x, f x)
\end{aligned}
$$

and

$$
\begin{aligned}
\int_{0}^{d(f x, f y)} \varphi(t) d t & =\left(\frac{x}{1+x}-\frac{y}{2}\right)^{2} \leq\left(\frac{x}{1+x}-\frac{1}{2} \cdot \frac{x}{1+x}\right)^{2} \\
& =\frac{1}{4}\left(\frac{x}{1+x}\right)^{2} \leq \alpha\left(\frac{x^{2}}{1+x}\right)\left(\frac{x^{2}}{1+x}\right)^{2} \\
& =\alpha\left(m_{1}(x, y)\right) \int_{0}^{m_{1}(x, y)} \varphi(t) d t
\end{aligned}
$$

Case $5.0 \leq y<\frac{x}{1+x}$ and $x \geq 1$. It is clear that

$$
\begin{aligned}
m_{1}(x, y) & =\max \left\{d(x, y), d(x, f x), d(y, f y), \frac{1}{2}[d(x, f y)+d(y, f x)]\right\} \\
& =\max \left\{x-y, \frac{x^{2}}{1+x}, \frac{y}{2}, \frac{1}{2}\left(x-\frac{y}{2}+\frac{x}{1+x}-y\right)\right\} \\
& =x-y=d(x, y)
\end{aligned}
$$

and

$$
\begin{aligned}
\int_{0}^{d(f x, f y)} \varphi(t) d t & =\left(\frac{x}{1+x}-\frac{y}{2}\right)^{2} \leq\left(\frac{x}{2}-\frac{y}{2}\right)^{2} \\
& =\frac{1}{4}(x-y)^{2} \leq \alpha(x-y)(x-y)^{2} \\
& =\alpha\left(m_{1}(x, y)\right) \int_{0}^{m_{1}(x, y)} \varphi(t) d t
\end{aligned}
$$

That is, the conditions of Theorem 3.3 are fulfilled. It follows from Theorem 3.3 that $f$ has a unique fixed point $0 \in X$ and $\lim _{n \rightarrow \infty} f^{n} x=0$ for each $x \in X$.

Remark 4.7 Theorem 3.4 extends Theorem 1.3. The following example shows that Theorem 3.4 both generalizes substantially Theorem 1.3 and differs from Theorem 1.5 . 
Example 4.8 Let $X=\mathbb{R}^{+}$be endowed with the Euclidean metric $d=|\cdot|, f: X \rightarrow X, \varphi$ : $\mathbb{R}^{+} \rightarrow \mathbb{R}^{+}$and $\alpha: \mathbb{R}^{+} \rightarrow[0,1)$ be defined by

$$
\begin{aligned}
& f(x)= \begin{cases}\frac{x}{2}, & \forall x \in[0,1], \\
x-1, & \forall x \in(1,+\infty),\end{cases} \\
& \varphi(t)=2 t, \quad \forall t \in \mathbb{R}^{+} \text {and } \alpha(t)= \begin{cases}\frac{1}{4}, & \forall t \in[0,1], \\
\frac{(t-1)^{2}}{t^{2}}, & \forall t \in(1,+\infty) .\end{cases}
\end{aligned}
$$

It is clear that $(\varphi, \alpha) \in \Phi \times \Phi_{3}$ and $O_{f}(u)$ is bounded for each $u \in X$. Let $x, y \in X$ with $y<x$. In order to verify (3.16), we have to consider four possible cases as follows:

Case $1.0 \leq y<x \leq 1$. Note that

$$
\begin{aligned}
m_{2}(x, y) & =\max \{d(x, y), d(x, f x), d(y, f y), d(x, f y), d(y, f x)\} \\
& =\max \left\{x-y, \frac{x}{2}, \frac{y}{2}, x-\frac{y}{2},\left|y-\frac{x}{2}\right|\right\} \\
& =x-\frac{y}{2}=d(x, f y)
\end{aligned}
$$

and

$$
\begin{aligned}
\int_{0}^{d(f x, f y)} \varphi(t) d t & =\left|\frac{x}{2}-\frac{y}{2}\right|^{2} \\
& =\frac{(x-y)^{2}}{4} \leq \frac{\left(x-\frac{y}{2}\right)^{2}}{4} \\
& =\alpha(d(x, f y)) \int_{0}^{d(x, f y)} \varphi(t) d t \\
& =\alpha\left(m_{2}(x, y)\right) \int_{0}^{m_{2}(x, y)} \varphi(t) d t .
\end{aligned}
$$

Case 2. $0 \leq y \leq 1<x \leq 1+\frac{y}{2}$. Clearly

$$
\begin{aligned}
m_{2}(x, y) & =\max \{d(x, y), d(x, f x), d(y, f y), d(x, f y), d(y, f x)\} \\
& =\max \left\{x-y, 1, \frac{y}{2}, x-\frac{y}{2},|y-x+1|\right\} \\
& =1=d(x, f x)
\end{aligned}
$$

and

$$
\begin{aligned}
\int_{0}^{d(f x, f y)} \varphi(t) d t & =\left|x-1-\frac{y}{2}\right|^{2} \\
& \leq\left(-\frac{y}{2}\right)^{2} \leq \frac{1}{4} \\
& =\alpha(d(x, f x)) \int_{0}^{d(x, f x)} \varphi(t) d t \\
& =\alpha\left(m_{2}(x, y)\right) \int_{0}^{m_{2}(x, y)} \varphi(t) d t .
\end{aligned}
$$


Case 3. $0 \leq y \leq 1$ and $1+\frac{y}{2}<x<+\infty$. Obviously

$$
\begin{aligned}
m_{2}(x, y) & =\max \{d(x, y), d(x, f x), d(y, f y), d(x, f y), d(y, f x)\} \\
& =\max \left\{x-y, 1, \frac{y}{2}, x-\frac{y}{2},|y-x+1|\right\} \\
& =x-\frac{y}{2}=d(x, f y)
\end{aligned}
$$

and

$$
\begin{aligned}
\int_{0}^{d(f x, f y)} \varphi(t) d t & =\left|x-1-\frac{y}{2}\right|^{2} \leq \frac{\left(x-1-\frac{y}{2}\right)^{2}}{\left(x-\frac{y}{2}\right)^{2}} \cdot\left(x-\frac{y}{2}\right)^{2} \\
& =\alpha(d(x, f y)) \int_{0}^{d(x, f y)} \varphi(t) d t \\
& =\alpha\left(m_{2}(x, y)\right) \int_{0}^{m_{2}(x, y)} \varphi(t) d t .
\end{aligned}
$$

Case 4. $1<y<x<+\infty$. It follows that

$$
\begin{aligned}
m_{2}(x, y) & =\max \{d(x, y), d(x, f x), d(y, f y), d(x, f y), d(y, f x)\} \\
& =\max \{x-y, 1,1, x-y+1,|y-x+1|\} \\
& =x-y+1=d(x, f y)
\end{aligned}
$$

and

$$
\begin{aligned}
\int_{0}^{d(f x, f y)} \varphi(t) d t & =(x-y)^{2} \leq \frac{(x-y+1-1)^{2}}{(x-y+1)^{2}} \cdot(x-y+1)^{2} \\
& =\alpha(d(x, f y)) \int_{0}^{d(x, f y)} \varphi(t) d t \\
& =\alpha\left(m_{2}(x, y)\right) \int_{0}^{m_{2}(x, y)} \varphi(t) d t .
\end{aligned}
$$

That is, the conditions of Theorem 3.4 are fulfilled. It follows from Theorem 3.4 that $f$ has a unique fixed point $0 \in X$ and $\lim _{n \rightarrow \infty} f^{n} u=0$ for each $u \in X$. But we do not invoke Theorems 1.3 and 1.5 to show the existence of a fixed point of $f$ in $X$.

Suppose that $f$ satisfies the conditions of Theorem 1.3, that is, there exists some $c \in(0,1)$ satisfying

$$
\begin{aligned}
(x-y)^{2} & =\int_{0}^{d\left(f x_{x} f y\right)} \varphi(t) d t \leq c \int_{0}^{m_{2}(x, y)} \varphi(t) d t \\
& =c(x-y+1)^{2}, \quad \forall x, y \in(1,+\infty) \text { with } y<x,
\end{aligned}
$$

which yields

$$
1=\lim _{x-y \rightarrow+\infty} \frac{(x-y)^{2}}{(x-y+1)^{2}} \leq c<1,
$$

which is impossible. 
Suppose that $f$ satisfies the conditions of Theorem 1.5, that is, there exist $\alpha, \beta: \mathbb{R}^{+} \rightarrow$ $[0,1)$ satisfying

$$
\alpha(t)+\beta(t)<1, \quad \forall t \in \mathbb{R}^{+}, \quad \limsup _{s \rightarrow 0^{+}} \beta(s)<1, \quad \limsup _{s \rightarrow t^{+}} \frac{\alpha(s)}{1-\beta(s)}<1, \quad \forall t>0
$$

and

$$
\begin{aligned}
1 & =(3-2)^{2}=\int_{0}^{d(f 3, f 2)} \varphi(t) d t \\
& \leq \alpha(d(3,2)) \int_{0}^{d(3, f 3)} \varphi(t) d t+\beta(d(3,2)) \int_{0}^{d(2, f 2)} \varphi(t) d t \\
& =\alpha(1) \cdot 1+\beta(1) \cdot 1=\alpha(1)+\beta(1),
\end{aligned}
$$

which means that

$$
1 \leq \alpha(1)+\beta(1)<1,
$$

which is absurd.

\section{Competing interests}

The authors declare that they have no competing interests.

\section{Authors' contributions}

All authors read and approved the final manuscript.

\section{Author details}

'Department of Mathematics, Liaoning Normal University, Dalian, Liaoning 116029, People's Republic of China.

²Department of Mathematics and RINS, Gyeongsang National University, Jinju, 660-701, Korea.

\section{Acknowledgements}

The authors would like to thank the referees for useful comments and suggestions. This research was supported by the Science Research Foundation of Educational Department of Liaoning Province (L2012380) and the fund of the Research Promotion Program, Gyeongsang National University, 2013 (RPP-2013-023).

Received: 15 January 2014 Accepted: 4 June 2014 Published: 18 July 2014

\section{References}

1. Aliouche, A: A common fixed point theorem for weakly compatible mappings in symmetric spaces satisfying a contractive condition of integral type. J. Math. Anal. Appl. 322(2), 796-802 (2006). doi:10.1016/j.jmaa.2005.09.068

2. Altun, I, Türkoğlu, D: Some fixed point theorems for weakly compatible mappings satisfying an implicit relation. Taiwan. J. Math. 13(4), 1291-1304 (2009)

3. Altun, I, Türkoǧlu, D, Rhoades, BE: Fixed points of weakly compatible maps satisfying a general contractive of integral type. Fixed Point Theory Appl. 2007, Article ID 17301 (2007). doi:10.1155/2007/17301

4. Beygmohammadi, M, Razani, A: Two fixed-point theorems for mappings satisfying a general contractive condition of integral type in the modular space. Int. J. Math. Math. Sci. 2010, Article ID 317107 (2010). doi:10.1155/2010/317107

5. Branciari, A: A fixed point theorem for mappings satisfying a general contractive condition of integral type. Int. J. Math. Math. Sci. 29(9), 531-536 (2002). doi:10.1155/S0161171202007524

6. Djoudi, A, Aliouche, A: Common fixed point theorems of Greguš type for weakly compatible mappings satisfying contractive conditions of integral type. J. Math. Anal. Appl. 329(1), 31-45 (2007). doi:10.1016/j.jmaa.2006.06.037

7. Djoudi, A, Merghadi, F: Common fixed point theorems for maps under a contractive condition of integral type. J. Math. Anal. Appl. 341(2), 953-960 (2008). doi:10.1016/j.jmaa.2007.10.064

8. Jachymski, J: Remarks on contractive conditions of integral type. Nonlinear Anal. 71(3-4), 1073-1081 (2009). doi:10.1016/j.na.2008.11.046

9. Kutbi, MA, Imdad, M, Chauhan, S, Sintunavarat, W: Some integral type fixed point theorems for non-self mappings satisfying generalized $(\psi, \varphi)$-weak contractive conditions in symmetric spaces. Abstr. Appl. Anal. 2014, Article ID 519038 (2014). doi:10.1155/2014/519038

10. Liu, Z, Li, X, Kang, SM, Cho, SY: Fixed point theorems for mappings satisfying contractive conditions of integral type and applications. Fixed Point Theory Appl. 2011, Article ID 64 (2011). doi:10.1186/1687-1812-2011-64 
11. Liu, Z, Li, ZL, Kang, SM: Fixed point theorems of contractive mappings of integral type. Fixed Point Theory Appl. 2013, Article ID 300 (2013). doi:10.1186/1687-1812-2013-300

12. Liu, Z, Lu, Y, Kang, SM: Fixed point theorems for mappings satisfying contractive conditions of integral type. Fixed Point Theory Appl. 2013, Article ID 267 (2013). doi:10.1186/1687-1812-2013-267

13. Mongkolkeha, C, Kumam, P: Fixed point and common fixed point theorems for generalized weak contraction mappings of integral type in modular spaces. Int. J. Math. Math. Sci. 2011, Article ID 705943 (2011). doi:10.1155/2011/705943

14. Sintunavarat, W, Kumam, P: Gregus-type common fixed point theorems for tangential multivalued mappings of integral type in metric spaces. Int. J. Math. Math. Sci. 2011, Article ID 923458 (2011). doi:10.1155/2011/923458

15. Sintunavarat, W, Kumam, P: Gregus type fixed points for a tangential multi-valued mappings satisfying contractive conditions of integral type. J. Inequal. Appl. 2011, Article ID 3 (2011). doi:10.1186/1029-242X-2011-3

16. Rhoades, BE: Two fixed-point theorems for mappings satisfying a general contractive condition of integral type. Int. J. Math. Math. Sci. 2003(63), 4007-4013 (2003). doi:10.1155/S0161171203208024

17. Suzuki, T: Meir-Keeler contractions of integral type are still Meir-Keeler contractions. Int. J. Math. Math. Sci. 2007, Article ID 39281 (2007). doi:10.1155/2007/39281

18. Vijayaraju, P, Rhoades, BE, Mohanraj, R: A fixed point theorem for a pair of maps satisfying a general contractive condition of integral type. Int. J. Math. Math. Sci. 2005(15), 2359-2364 (2005). doi:10.1155/JMMS.2005.2359

doi:10.1186/1687-1812-2014-138

Cite this article as: Liu et al.: Existence and approximations of fixed points for contractive mappings of integral type. Fixed Point Theory and Applications 2014 2014:138

\section{Submit your manuscript to a SpringerOpen ${ }^{\circ}$ journal and benefit from:}

- Convenient online submission

- Rigorous peer review

- Immediate publication on acceptance

- Open access: articles freely available online

- High visibility within the field

- Retaining the copyright to your article 